\title{
UCR RADIOCARBON DATES I
}

\author{
R E TAYLOR \\ Department of Anthropology, \\ Center for Social and Behavioral Science Research, \\ Institute of Geophysics, University of California, \\ Riverside, California 92502
}

\begin{abstract}
A radiocarbon facility has been installed at the University of California, Riverside (UCR) to support interdisciplinary studies including archaeologic, archaeometric, geophysical, and geologic research. The laboratory was built between 1970 and 1973. Initially, a sample pretreatment and combustion system designed for a proportional $\mathrm{CO}_{2}$ counting system was installed. It was designed after concepts developed at the University of California Los Angeles (UCLA) and New Zealand (Institute of Nuclear Sciences) Laboratories, and began processing samples in November 1972.

A $1.7 \mathrm{~L} \mathrm{CO}_{2}$ proportional counter was then installed in the UCR laboratory which commenced operation in late September, 1973. The counting system was assembled from components originally fabricated by $G \mathrm{~J}$ Fergusson. Calculated radiocarbon ages are based on a $5568 \mathrm{yr}$ half-life as recommended by the Eighth International Radiocarbon Dating Conference, October 1972, Lower Hutt, New Zealand. All samples are counted at a filling pressure equivalent to $76 \mathrm{~cm}$ at $20^{\circ} \mathrm{C}$. The standard for the contemporary biosphere is 0.95 NBS oxalic acid and AD 1950 constitutes the zero reference year. Statistical errors are calculated by combining the standard $1 \sigma$ standard deviations of the background and sample counts. Samples which approach modern or background values are reported with $2 \sigma$ limits. All samples were subjected to accepted $\mathrm{HCl}$, $\mathrm{NaOH}$ or other special chemical pretreatments depending on specific conditions to exclude contamination.

Through the cooperation of W F Libby and Rainer Berger, the Isotope Laboratory of the Institute of Geophysics at UCLA performed radiocarbon determinations on sample gases prepared by the UCR facility for calibration and interlaboratory checks. UCR I contains the interlaboratory calibration data (Table 1) and sample descriptions for joint UCLA/UCR measurements. UCR II and subsequent lists will contain radiocarbon dates measured exclusively at the Riverside laboratory.
\end{abstract}

\section{ACKNOWLEDGMENTS}

The author wishes to express his gratitude to those who contributed to the development of the UCR radiocarbon facility. W F Libby and Rainer Berger provided continuing equipment support, advice, and encouragement. J G Fergusson, Scientific Research Equipment Corp of Baltimore, Maryland, provided technical assistance. $H$ Suess and Donald Sullivan, Radiocarbon Laboratory, Scripps Inst of Oceanography, University of California, San Diego, provided equipment and expert assistance. Operational support came from various sources including the 
American Philosophical Society, UCR Intramural Research Grants, the Graduate Division at UCR, and a Dept of Labor grant to UCR. The dedicated laboratory work of Peter Slota, Dwight Sawyer, and Stanley Sheldon is also very much appreciated. The support and encouragement of James Earley and Michael Reagan, College of Social and Behavioral Sciences, and Alan Beals and Sylvia Broadbent, Anthropology Dept, is gratefully acknowledged.

TABLE 1

Interlaboratory calibration samples

\begin{tabular}{cccc}
\hline $\begin{array}{c}\text { UCR } \\
\text { sample no }\end{array}$ & Date & \multicolumn{1}{c}{$\begin{array}{c}\text { UCLA } \\
\text { sample no. }\end{array}$} & Date \\
\hline UCR-125* & modern $(<100 \mathrm{yr})$ & UCLA-1887 & modern $(<100 \mathrm{yr})$ \\
UCR-129* & $180 \pm 100$ & UCLA-1888 & $190 \pm 60$ \\
UCR-119F & $720 \pm 100$ & UCLA-1867F & $770 \pm 80$ \\
UCR-128* & $940 \pm 100$ & UCLA-1892 & $1170 \pm 80$ \\
UCR-118D & $1580 \pm 150$ & UCLA-1800D & $1650 \pm 80$ \\
UCR-126* & $3750 \pm 100$ & UCLA- 900 & $3760 \pm 80$ \\
\hline
\end{tabular}

* Descriptions for these samples will appear in UCR II.

\section{SAMPLE DESCRIPTIONS}

\section{ARCHAEOLOGIC SAMPLES}

\section{Perris Reservoir series, Riverside Co, California}

Studies were conducted by the Archaeol Research Unit, UCR, as salvage excavations supported by California Dept of Parks and Recreation, Perris Reservoir, Riverside Co, California $\left(33^{\circ} 50^{\prime} \mathrm{N}, 117^{\circ} 30^{\prime} \mathrm{W}\right)$. This site represents the most intensely studied late prehistoric archaeologic region in interior Southern California. The results of the excavations will appear elsewhere (O'Connell, et al). Samples were coll Spring 1971 and subm by $P$ Wilke and T King, UCR.

\section{UCR-101/UCLA-1815. Peppertree site}

AD 1080

Charcoal from Feature 9, a hearth underlying primary living floor in Area 2 of 4-Riv-463, Peppertree site. Comment (PW): dates period prior to beginning of intensive site occupation.

\section{UCR-102/UCLA-1816. Peppertree site}

$215 \pm 60$

Charcoal from Feature 4, a hearth overlying primary living floor in Area 2 of 4-Riv-463, Peppertree site. Comment (TK): living floor represents period of most intensive use of site.

\section{UCR-103/UCLA-1817. Peppertree site}

$2200 \pm 80$

Charcoal from Feature 14, a hearth, basal feature of site, in otherwise generally sterile sand of 4-Riv-463, Peppertree site. Comment (TK): date is compatible with other evidence of occupation of the reservoir area 
during the period ca 2000 BC. Other evidence includes an Elko CornerNotched projectile point from the Charles Mott site (4-Riv-464).

\section{UCR-104/UCLA-1818. Charles Mott site}

$210 \pm 60$

Charcoal from Feature 13, a hearth from Loc 1, Unit 2S/18W, with cottonwood Triangular concave-base projectile point and Tizon Brown ware. Comment (PW): dates period of most intensive site use.

UCR-105/UCLA-1819. Dead Dog site

Charcoal from Loc B, Unit 7, a hearth assoc with small triangular projectile points and worked valves (Argopecten sp). Comment (TK): although it is not impossible that site was in use as late as AD 1800, date is the only evidence for it.

\section{Buchanan Reservoir series, Madera Co, California}

Human burials excavated from a mortuary complex in salvage operation in Buchanan Reservoir region ( $\left.37^{\circ} 13^{\prime} \mathrm{N}, 119^{\circ} 59^{\prime} \mathrm{E}\right)$. Significance of data will be discussed elsewhere. All dates were obtained on collagen component of bone after the method of Berger et al, (1964). Coll 1972 by T King, UCR.

\section{UCR-118A/UCLA-1860A. Jones site}

$$
1690 \pm 100
$$

Bone collagen from Human Burial 52, Jones site (4-Mad-159) on bedrock under $1 \mathrm{~m}$ midden.

UCR-118B/UCLA-1860B. Schwabacker site

Bone collagen from Human Burial 44, Schwabacker site (4-Mad-117) on bedrock under $70 \mathrm{~cm}$ midden.

UCR-118C/UCLA-1860C. Schwabacker site

$$
1470 \pm 100
$$

Bone collagen from Human Burial 59, Schwabacker site (4-Mad-117) on bedrock under $70 \mathrm{~cm}$ midden.

\section{UCR-118D/UCLA-1860D. Dancing Cow site}

$$
1650 \pm 80
$$

Bone collagen from Human Burial 24, Dancing Cow site (4-Mad106) on bedrock under $1.5 \mathrm{~m}$ midden.

UCR-118E/UCLA-1860E. Dancing Cow site

$$
1740 \pm 100
$$

Bone collagen from Human Burial 11, Dancing Cow site (4-Mad 106).

UCR-118F/UCLA-1860F. Schwabacker site

$$
970 \pm 80
$$

Bone collagen from Human Burial 21, Schwabacker site (4-Mad-117). 
UCR-118G/UCLA-1860G. Schwabacker site

$1745 \pm 100$

Bone collagen from Human Burial 16, Schwabacker site (4-Mad-117).

UCR-118H/UCLA-1860H. Jones site

$1400 \pm 90$

Bone collagen from Human Burial 13, Jones site (4-Mad-159) on bedrock under $1 \mathrm{~m}$ midden.

UCR-118I/UCLA-1860I. Dancing Cow site

$1010 \pm 80$

Bone collagen from Human Burial 33, Dancing Cow site (4-Mad-106) on bedrock under $1.5 \mathrm{~m}$ midden.

UCR-118J/UCLA-1860J. Jones site

$1540 \pm 110$

Bone collagen from Human Burial 5, Jones site (4-Mad-159) on bedrock under $1 \mathrm{~m}$ midden.

UCR-118K/UCLA-1860K. Schwabacker site

$1310 \pm 80$

Bone collagen from Human Burial 20, Schwabacker site (4-Mad-117) on bedrock under $70 \mathrm{~cm}$ midden.

UCR-118L/UCLA-1860L. Schwabacker site

$1305 \pm 80$

Bone collagen from Human Burial 8, Schwabacker site (4-Mad-117) on bedrock under $70 \mathrm{~cm}$ midden.

UCR-118N/UCLA-1860N. Schwabacker site

$1425 \pm 80$

Bone collagen from Human Burial 36, Schwabacker site (4-Mad-117) on bedrock under $70 \mathrm{~cm}$ midden.

UCR-1180/UCLA-18600. Dancing Cow site

$2000 \pm 80$ 50 BC

Bone collagen from Human Burial 1, Dancing Cow site (4-Mad-106) on bedrock under $1.5 \mathrm{~m}$ midden.

UCR-118P/UCLA-1860P. Jones site

$1630 \pm 80$

Bone collagen from Human Burial 6, Jones site (4-Mad-159) on bedrock under $1 \mathrm{~m}$ midden.

\section{El Morro Canyon series}

Samples found during excavations conducted by California State Univ, Fullerton, in El Morro Canyon $\mathrm{N}$ of Laguna Beach, Orange Co, California $\left(33^{\circ} 34^{\prime} 30^{\prime \prime} \mathrm{N}, 117^{\circ} 48^{\prime} 30^{\prime \prime} \mathrm{W}\right)$. Coll Spring 1972 by J L Zahniser, Dept Anthropol, California State Univ, Fullerton.

\section{UCR-119A/UCLA-1867A. El Morro Canyon}

$3500 \pm 100$

Charcoal from Unit W-45-B, 40 to $50 \mathrm{~cm}$ below datum at Site 4 -Ora327, N side of El Morro Canyon. Comment (JZ): unexpectedly early date. 
UCR-119B/UCLA-1867B. El Morro Canyon

Marine shell from Unit N1-C, 0 to $10 \mathrm{~cm}$ below datum at Site 4-Ora327, N side of El Morro Canyon.

UCR-119C/UCLA-1867C. El Morro Canyon

$570 \pm 80$

Marine shell from Unit N1-B-C-D, 30 to $50 \mathrm{~cm}$ below datum at Site 4-Ora-327, N side of El Morro Canyon.

\section{Rincon Valley series, Arizona}

Samples are from excavations in habitation areas in 2 sites on $\mathrm{N}$ side of lower Rincon Valley, E of Tucson, Arizona. Samples were coll 1964 by J L Zahniser.

\section{UCR-119D/UCLA-1867D. Loma Alta site}

$890 \pm 70$

Charred roof timber from Unit $1, \mathrm{Rm} \mathrm{3,} \mathrm{E} \mathrm{half} \mathrm{from} \mathrm{probable} \mathrm{roof}$ fall at Loma Alta site $\left(32^{\circ} 8^{\prime} \mathrm{N}, 110^{\circ} 43^{\prime} \mathrm{W}\right)$. Comment (JZ): radiocarbon date is ca $200 \mathrm{yr}$ earlier than anticipated-early Tanque Verde phase, AD 1100 to 1300. See Zahniser (1966).

\section{UCR-119E/UCLA-1867E. Site BB:14}

$1065 \pm 80$

Charred corn (Zea maize) from lower fill of House No. 4, at Site $\mathrm{BB}: 14\left(32^{\circ} 8^{\prime} 20^{\prime \prime} \mathrm{N}, 110^{\circ} 43^{\prime} \mathrm{W}\right)$. Fill material probably originally stored in ceramic vessels on roof of house (Zahniser, 1966). Comment (JZ): radiocarbon date is ca $200 \mathrm{yr}$ earlier than anticipated date-early Tanque Verde phase, AD 1100 to 1300.

\section{UCR-119F/UCLA-1867F. Loma Alta site}

$\mathbf{7 7 0} \pm \mathbf{8 0}$

Charred roof timber from Unit 3, Rm 2, top $50 \mathrm{~cm}$ fill, probably roof fall from Loma Alta site $\left(32^{\circ} 8^{\prime} \mathrm{N}, 110^{\circ} 39^{\prime} \mathrm{W}\right)$.

\section{Earthquake Studies series}

II. GEOLOGIC SAMPLES

Geologic studies of a portion of the San Jacinto fault zone near San Bernardino, California were made to locate more precisely the most recently active fault trace(s) within the zone, and to determine the nature and dates of the most recent fault movements (Elders, 1973). Samples were obtained from $O$ Huber, California Dept Water Resources, Palmdale, California (UCR-106-109) and a team of undergraduate students, Univ California, Riverside (UCR-110-117) funded under Student-oriented Studies Program, Nat Sci Foundation (GY-9667). Samples were coll JuneSeptember 1972 by O Huber and K Sieh and subm by K Sieh, Dept Geol, Univ California, Riverside.

$$
\begin{array}{lll}
\text { UCR-106/UCLA-1826. } & \begin{array}{l}
\text { Santa Ana Valley } \\
\text { Pipeline }
\end{array} & \begin{array}{c}
\text { 300 } \\
\text { AD 1650 }
\end{array} \\
\text { Wood from channel alluvium at Pipeline Sta } & 182+60 \text {, at depth }
\end{array}
$$


$5.2 \mathrm{~m}\left(34^{\circ} 04^{\prime} \mathrm{N}, 117^{\circ} 18^{\prime} \mathrm{E}\right)$. Comment $(\mathrm{OH})$ : no direct evidence that deposit was offset by San Jacinto Fault.

\section{UCR-107/UCLA-1827. Santa Ana Valley $\quad 100 \pm 80$ Pipeline AD 1850}

Wood from channel alluvium at Pipeline Sta $179+75$, at depth $4.6 \mathrm{~m}\left(34^{\circ} 04^{\prime} \mathrm{N}, 117^{\circ} 18^{\prime} \mathrm{E}\right)$. Comment $(\mathrm{OH})$ : no direct evidence that deposit was offset by San Jacinto Fault.

UCR-108/UCLA-1828. Santa Ana Valley $\quad 33,000 \pm 900$ Pipeline 31,050 BC

Wood from deformed, tilted, gravelly clay, offset by San Jacinto Fault at Pipeline Sta $172+15$, at depth $6.7 \mathrm{~m}\left(34^{\circ} 04^{\prime} \mathrm{N}, 117^{\circ} 18^{\circ} \mathrm{E}\right)$.

UCR-109/UCLA-1832. Santa Ana Valley Pipeline

Wood from iron-stained gravelly sand, offset by San Jacinto Fault at Pipeline Sta $174+25$ at depth $5.8 \mathrm{~m}\left(34^{\circ} 04^{\prime} \mathrm{N}, 117^{\circ} 18^{\prime} \mathrm{E}\right)$.

\section{UCR-110/UCLA-1836. Barton Rd}

$1500 \pm 150$

Wood from root, $7.6 \mathrm{~cm}$ diam, coll $1.2 \mathrm{~m}$ from ground surface in silty clay in Barton Rd, Trench $3\left(34^{\circ} 03^{\prime} \mathrm{N}, 117^{\circ} 17^{\prime} \mathrm{E}\right)$.

\section{UCR-111/UCLA-1837. Barton Rd}

$1670 \pm 150$

Black, carbonaceous organic residue and peat from $1.5 \mathrm{~m}$ area, $1.5 \mathrm{~m}$ to $1.8 \mathrm{~m}$ from surface in fine sand alternating with silt from Barton $\mathrm{Rd}$, Trench $3\left(34^{\circ} 03^{\prime} \mathrm{N}, 117^{\circ} 17^{\prime} \mathrm{E}\right)$.

\section{UCR-113/UCLA-1839. Barton Rd}

$$
2460 \pm 160
$$

Black, carbonaceous organic residue from area $2.4 \mathrm{~m}$, ca $2.4 \mathrm{~m}$ below ground in silty clay from Barton $\mathrm{Rd}$, Trench $2\left(34^{\circ} 03^{\prime} \mathrm{N}, 117^{\circ} 17^{\prime} \mathrm{E}\right)$.

\section{UCR-114/UCLA-1840. Walnut St}

$$
\begin{aligned}
& 4400 \pm 250 \\
& 2450 \mathrm{BC}
\end{aligned}
$$

Reed in black, carbonaceous, organic matrix from $2.4 \mathrm{~m}$ zone, $2.4 \mathrm{~m}$ below ground in an intraformational breccia from Walnut St Trench $\left(34^{\circ} 04^{\prime} \mathrm{N}, 117^{\circ} 19^{\prime} \mathrm{E}\right)$.

UCR-115/UCLA-1841. Walnut St

$$
3180 \pm 150
$$

$$
1230 \mathrm{BC}
$$

Wood fragments and black, carbonaceous, organic material from 2.4 to $2.7 \mathrm{~m}$ below ground in light gray silty sand from Walnut St Trench $\left(34^{\circ} 05^{\prime} \mathrm{N}, 117^{\circ} 19^{\prime} \mathrm{E}\right)$.

UCR-116/UCLA-1842. Barton Rd

$$
670 \pm 80
$$

Fresh water gastropods (Limnaea adeline Tryon, Physa gabbi Tryon, Planorbis trivolvis Say, Succinea oregonensis Lea) from area $6 \mathrm{~m}$, 
$12 \mathrm{~m}$ below surface in silty clay from Barton $\mathrm{Rd}$, Trench $2\left(34^{\circ} 04^{\prime} \mathrm{N}\right.$, $\left.117^{\circ} 17^{\prime} \mathrm{E}\right)$.

\section{UCR-117 / UCLA-1843. Walnut St}

$5540 \pm 200$

sand in Walnut St Trench $\left(34^{\circ} 05^{\prime} \mathrm{N}, 117^{\circ} 19^{\prime} \mathrm{E}\right)$.

General Comment (ET): except for UCR-115, ages fall into stratigraphic order and provide a preliminary date for prior movements along fault zones.

\section{REFERENCES}

Berger, R, Horney, A G, and Libby, W F, 1964, Radiocarbon dating of bone and shell from their organic components: Science, v 144, p 999-1001.

Elders, W A, (ed), 1973, Geological investigations of the San Jacinto Fault Zone and aspects of the socio-economic impact of earthquakes in the Riverside-San Bernardino Area, California: Univ California, Riverside campus mus contr, no. 3,
Riverside.

O'Connell, J F, et al, (eds), Perris Reservoir: late pre-historic demographic change in southeastern California: Archaeol repts, State of California, Dept Parks and Recre-
ation, Sacramento, in press.

Zahniser, J L, 1966, Late prehistoric villages southeast of Tucson, Arizona and the archaeology of the Tanque Verde phase: The Kiva, v 31, no. 3, p 1-11. 